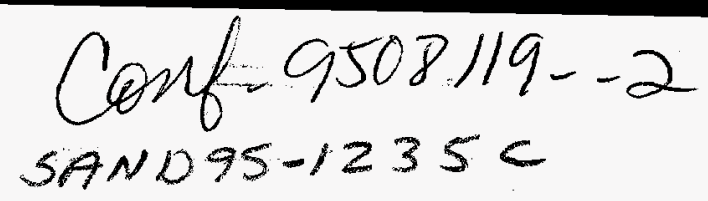

\title{
Current Status and Future Research in Motion Planning *
}

\author{
Yong K. Hwang \\ Sandia National Laboratories \\ Albuquerque, New Mexico 87185
}

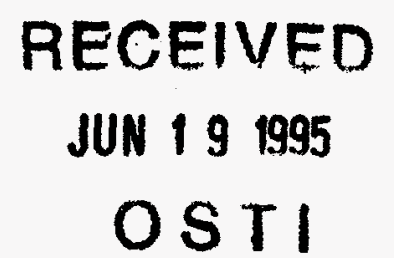

\begin{abstract}
There have been numerous research efforts in the field of motion planning, resulting in many theoretical and practical results. We review the current status of existing motion planning algorithms, evaluate their completenesses and efficiencies on modern computers, and suggest fruitful future research directions.
\end{abstract}

\section{Introduction}

Motion planning usually refers to the problem of finding a short, collision-free motion of a robot performing a given task. A majority of research considers the task of moving the robot from one configuration to another, or tracing a curve with the robot tool tip. The motion planning problem is inherently complex, and the best known complete algorithm is exponential in the number of degrees of freedom (dof) and polynomial in the geometric complexity of the robot and obstacles [3]. For low-dof problems, e.g., a mobile robot in the plane with 3 dof, efficient and complete algorithms exist. For a robot with 6 dof or more, e.g., an industrial robot or a rigid object in 3-dimensional space, a complete algorithm that also runs efficiently (a few minutes on a workstation) is not quite available. Consequently, many fast heuristic motion planners have been developed at the expense of losing completeness, i.e., no guarantee of solutions. Each motion planning application demands a different balance of efficiency and completeness. For example, in off-line programming of robots in manufacturing environments, many hours of computation time can be used to find an optimal robot motion. In interactive robotic operations, on the other hand, a fast, incomplete planner is preferred since interactive operations demand fast response time and the human operator can manually move the robot in case the motion planner cannot find a path.

We will review the recent work in motion planning in two categories: rigid objects and manipulators. For each category, the efficiencies and completeness of algorithms are discussed followed by suggestions for future research.

${ }^{*}$ This work has been performed at Sandia National Laboratories and supported by the U.S. Department of Energy under Contract DE-AC04-94AL85000.

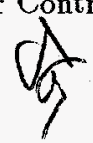

DISTRIBUTION

\section{Rigid objects}

Autonomous navigation of omni-directional mobile robots requires a motion planner for a rigid body. This type of motion planner is also needed to plan part mo tions in assembly planning. For 2D problems where the robot has 3 dof, a brute force method based on a grid suffices. In [12], such a method is implemented on a graphics hardware, and the computation times are in tens of seconds. We believe that with the memory capacity and CPU speed of a present workstation, this approach can be used for problems up to 4 dof. For 3 D problems where dof is 6 , two efficient algorithms that also guarantee a solution are $[1,8]$. The algorithm in [1] is a potential-field approach combined with a random subgoal generation to escape local minima, and is probabilistically complete. In [8], a resolutioncomplete motion planner is developed using a search strategy called Sandros, which can be characterized as a search on a dynamic graph of subgoals that are iteratively refined during the search. Both of the above algorithms take a few minutes to solve moderately hard problems, and take longer as problems get harder.

The motion planning of parts in mechanical assemblies requires two algorithms: one for motion with contact, and one for motion without. Motion without contact can be planned with a gross motion planner such as those described above, but the motion in contact needs different approaches such as the more rigorous construction of configuration-space obstacles [2] or polyhedral convex cones [5]. Algorithms to find feasible infinitesimal motion directions of a part in 6 dimensions ( 3 translation, 3 orientation) are developed in [7], and an algorithm for multiple extended translational motion is presented in [6].

The motion planning for 2D under perfect information is well studied from the practical point of view, as the present computer is fast enough for a bruteforce method. The current trend is incorporating sensing/sensor planning with motion planning. For 3 dimensions with 6 dof, a more efficient planner than those in $[1,8]$ is needed. Although it will be hard to develop a planner that performs uniformly better on all problems, fast application-specific algorithms can be developed within that domain. For planning of motion in contact for assembly, it remains to develop a planner that can generate multi-step translations and rotations. Since full 6 -dof motions are typically with- 


\section{DISCLAIMER}

Portions of this document may be illegible in electronic image products. Images are produced from the best available original document. 


\section{DISCLAIMER}

This report was prepared as an account of work sponsored by an agency of the United States Government. Neither the United States Government nor any agency thereof, nor any of their employees, make any warranty, express or implied, or assumes any legal liability or responsibility for the accuracy, completeness, or usefulness of any information, apparatus, product, or process disclosed, or represents that its use would not infringe privately owned rights. Reference herein to any specific commercial product, process, or service by trade name, trademark, manufacturer, or otherwise does not necessarily constitute or imply its endorsement, recommendation, or favoring by the United States Government or any agency thereof. The views and opinions of authors expressed herein do not necessarily state or reflect those of the United States Government or any agency thereof. 
out contact, it suffices for a planner to generate contact motions consisting of a sequence of primitive motions such as translation, rotation, helical motion, etc.

\section{Manipulators}

Since most manipulators have 5 to 7 dof, we will concentrate on 6-dof manipulators here. For point-topoint movement problem for a manipulator, the first complete motion planner is presented in [13], which builds 2D slices of the joint-space obstacles. Although complete, this algorithm takes several seconds for a 2-dof problem, and is likely take tens of minutes for 6dof problems. More efficient algorithms are developed in $[10,4]$, which are again probabilistically and resolution complete, respectively. Both algorithms have the characteristic that they solve easy problems fast, and harder problems with a gradual increase of computation time. It is our view that markedly faster algorithms than those in $[10,4]$ are possible with the use of a massively-parallel or knowledge-based approach. For manipulators with 4 dof or less, a brute force method is sufficient, whereas for robots with dof greater than say 10 , it is better to plan a path for the manipulator hand and let the links follow the hand like a snake [14].

For collision avoidance of a manipulator tracing a curve with its tool tip, many local planners have been developed which use the redundancy of the manipulator to avoid obstacles and singularities or increase dexterity measures [11]. These algorithms are developed for on-line, real-time applications, and the computation times are typically in milliseconds. For offline applications, global algorithms to avoid obstacles while tracing a curve are presented in $[15,9]$. 'These algorithms can generate a motion to trace a curve that is heavily obscured by obstacles, and take minutes to tens of minutes of computation time. It is our view that for local planning the mathematical tools for realtime inverse kinematics are well established, and better methods for compensating sensor and model errors are needed. Incorporating process-related constraints is another interesting research direction. For global planning, the algorithms in $[15,9]$ seem sufficiently complete and general for off-line applications.

\section{Conclusions}

Motion planning is one of the more mature research fields in robotics. Practical algorithms exist for some problems, while faster algorithms with more understood performance are needed for problems with 6 dof or more. We should always compare the available compute power with the algorithm sophistication, since more problems are solvable with brute force methods as compute power increases.

\section{References}

[1] Barraquand, J. and Latombe, J.C., "A Monte-Carlo Algorithm for Path Planning with Many Degrees of
Freedom," Proc. of IEEE Int. Conf. on Robotics and Automation, pp. 1712-1717, Cincinnati, OH, 1990.

[2] Brost, R.C., "Dynamic analysis of planar manipulation tasks," Proc. of IEEE Int. Conf. on Robotics and Automation, pp. 2247-2254, Nice, France, May 1992.

[3] Canny, J.F., The Complexity of Robot Motion Planning, The MIT Press, Cambridge, MA, 1988.

[4] Chen, P.C. and Hwang, Y.K., "SANDROS: A Motion Planner with Performance Proportional to Task Difficulty," Proc. of IEEE Int. Conf. on Robotics and Automation, pp. 2346-2353, Nice, France, 1992.

[5] Goldman, A.J. and Tucker, A.W., "Polyhedral convex cones," in Kuhn, H.W. and Tucker, A.W. (eds.): Linear Inequalities and Related Systems. Annals of Mathematics Studies 38, Princeton, NJ: Princeton University Press, pp. 19-39.

[6] Halperin, D. and Wilson, R., "Assembly partitioning along simple paths: the case of multiple translations," Proc. of IEEE Int. Conf. on Robotics and Automation, Nagoya, Japan, 1995.

[7] Hirukawa, H., Matsui, T. and Takase, K., “A general algorithm for derivation and analysis of constrained motion of polyhedra in contact," Proc. of Int. Workshop on Intel. Robots and Systems, pp. 38-43, 1991.

[8] Hwang, Y.K. and Chen, P.C., "A Heuristic and Complete Planner for the Classical Mover's Problem," Proc. of IEEE Int. Conf. on Robotics and Automation, Nagoya, Japan, May, 1995.

[9] Hwang, Y.K., Chen, P.C., Maciejewski, A.A. and Neidigk, D.D., "A global motion planner for curve tracing robots," Proc. of IEEE Int. Conf. on Robotics and Autcmation, pp. 662-667, San Diego, CA, 1994.

[10] Kavraki, L. and Latombe, J.C., "Randomized preprocessing of configuration space for fast path planning," Proc. of IEEE Int. Conf. on Robotics and Automation, pp. 2138-2145, San Diego, CA, 1994.

[11] Klein, C., Chu-Jenq, C. and Ahmed, S., "Use of an extended Jacobian method to map algorithmic singularities," Proc. of IEEE Int. Conf. on Robotics and Automation, pp. 632-637, Atlanta, GA, 1993.

[12] Lengyel, J., Reichert, M., Donald, B.R. and Greenberg, D.P., "Real-Time Robot Motion Planning Using Rasterizing Computer Graphics Hardware," Computer Graphics, vol. 24, no. 4, pp. 327-335, Aug. 1990.

[13] Lozano-Perez, T., "A Simple Motion-Planning Algorithm for General Robot Manipulators," IEEE Journal of Robotics and Automation, vol. RA-3, no. 3, pp. 224-238, 1987.

[14] Maciejewski, A.A. and Klein, C.A., "Obstacle avoidance for kinematically redundant manipulators in dynamically varying environments," Int. Journal of Robotics Research, vol. 4, no. 3, pp. 109-117, 1985.

[15] Wenger, P., Chedmail, P. and Reynier, F., "A global analysis of following trajectories by redundant manipulators in the presence of obstacles," Proc. of IEEE Int. Conf. on Robotics and Automation, pp. 901-906, Atlanta, GA, 1993. 